\title{
ANÁLISIS DE LOS DESTINOS TURÍSTICOS DEL FUTURO
}

Valentina Barragán Acero

Escuela de Negocios, Gestión y Sostenibilidad

Programa Tecnología en Gestión de Servicios para Aerolíneas vabarragan2@poligran.edu.co

Marly Alejandra Bonilla Arias

Escuela de Negocios, Gestión y Sostenibilidad

Programa Tecnología en Gestión de Servicios para Aerolíneas mabonilla9@poligran.edu.co

Juliana Gómez Arias

Escuela de Negocios, Gestión y Sostenibilidad Programa Tecnología en Gestión de Servicios para Aerolíneas jugomeza1@poligran.edu.co

Ana Gabriela Martínez Pérez

Escuela de Negocios, Gestión y Sostenibilidad Programa Tecnología en Gestión de Servicios para Aerolíneas anmartinez49@poligran.edu.co

Karen Natalia Moreno Millán

Escuela de Negocios, Gestión y Sostenibilidad Programa Tecnología en Gestión de Servicios para Aerolíneas kamoreno24@poligran.edu.co

Valentina Navarrete Acuña

Escuela de Negocios, Gestión y Sostenibilidad Programa Tecnología en Gestión de Servicios para Aerolíneas vanavarrete@poligran.edu.co

David Leonardo Pérez Vargas

Escuela de Negocios, Gestión y Sostenibilidad Programa Tecnología en Gestión de Servicios para Aerolíneas daperezv@ poligran.edu.co

Isaac Mark Mclaughlin Silva Ramírez

Escuela de Negocios, Gestión y Sostenibilidad Programa Tecnología en Gestión de Servicios para Aerolíneas issilvar@poligran.edu.co

Elisabel Catalina Villalba Pérez

Escuela de Negocios, Gestión y Sostenibilidad Programa Tecnología en Gestión de Servicios para Aerolíneas elvillalba@poligran.edu.co 


\section{Resumen}

Actualmente, el mayor reto del mercado turístico es cubrir la demanda de viajeros que buscan nuevos destinos turísticos, y que a la vez desean experimentar una innovación tecnológica en ellos. El turismo espacial, se ha convertido en uno de los sectores más llamativos pertenecientes a la industria turística, y por ello las empresas relacionadas a este campo buscan satisfacer las futuras necesidades de los clientes a quienes van dirigidas sus actividades, e implementar un nuevo modelo de viaje que trabaje con los avances tecnológicos para así conocer el espacio exterior. En este artículo se analiza el impacto de las formas innovadoras de viaje actuales, y las que próximamente serán establecidas, basándose en los factores de brindar comodidad, seguridad y una experiencia completamente nueva a los clientes sin desaprovechar las nuevas tecnologías, para así lograr el reconocimiento del avance de la industria turística. Los resultados preliminares de esta investigación permiten evidenciar el número de proyectos que buscan ser implementados y lanzados al público, con relación a los destinos turísticos en el espacio exterior, y la posibilidad de lograr salir de este planeta de forma más asequible para los viajeros alrededor del mundo.

Palabras Clave: Mercado Turístico, Destinos Turísticos, Turismo Espacial, Viajes espaciales. 


\section{Introducción}

Gracias a los avances tecnológicos de los viajes que se han realizado hasta el momento, para nadie es un secreto que cada vez el usuario exige más, quiere conocer más lugares donde la experiencia sea única, que no sean los mismos destinos, que ya por tanta publicidad no se puedan disfrutar. Ahora en adelante, gracias a los adelantos tecnológicos, no solo es para que las personas conozcan sitios turísticos, sino que se vayan haciendo la idea de poder vivir en años futuros fuera del planeta Tierra.

Uno de los grandes pasos que ha dado el hombre es llegar a la Luna, los astronautas NEIL ARMSTRONG, MICHAEL COLLINS y BUZZ ALDRIN, en la misión Apolo XI, desde entonces compañías que cuentan con un personal capacitado, los cuales estudian los temas del espacio, han ido creando equipos innovadores. El ser humano tiene la necesidad de conocer nuevos destinos, la innovación en los viajes es cada vez más posible, los viajes a destinos no terrestres también, debido a las nuevos métodos o equipos que se han estado desarrollando al paso de los años por organizaciones desarrolladoras del turismo espacial, las cuales quieren mejorar la experiencia, comodidad y seguridad del pasajero, llevándolos a nuevos lugares turísticos, ya que antes eran inalcanzables, utilizando herramientas tecnológicas, estas ayudarán a llevar a los usuarios a nuevos lugares poco frecuentados, por el momento, las aerolíneas y agencias de viajes buscan cómo transportar más pasajeros a lo largo del mundo, incluso fuera de él y con esta evolución que ha llevado las entidades pioneras, los nuevos destinos desconocidos e interesantes para el ser humano en este sector serán importantes de presenciar en años futuros.

A partir de estos avances en el sector, en este documento muestra un avance que exhibirá esta industria en un futuro cercano. La forma en que se desarrolla esta investigación, es a partir de una revisión documental, en la cual se evidencia las mejoras que se desarrollarán en término de turismo espacial, en la cual se hablará, qué está pasando en el presente y lo que se generará en un futuro.

Además se indagó sobre las empresas: NASA, SpaceX, Virgin Galactic, Blue Origin y Orion Span, de cómo han llevado este tipo de proyectos en relación al turismo espacial, mostrando evolución, y que están teniendo en cuenta las entidades pioneras para la realización de este tipo de turismo; las cuales van a satisfacer este lujo (viajar) y ofrecer nuevas experiencias a los turistas, ya que también el hecho de cruzar nuevas fronteras y poder vivir en estas, son experiencias únicas y además posibles oportunidades afuera del Planeta y experiencias satisfactorias al pasajero. 


\section{Revisión Literaria}

"El turismo espacial es una modalidad de turismo en el que se realiza el viaje a más de 100 kilómetros de altura de la Tierra, lo que se considera la frontera del espacio" (Serrano Navalon \& Ramón Fernández, 2013) se puede evidenciar que en este tipo de turismo influyó mucho el sector privado ya que en su mayoría son los encargados de financiar cada uno de estos proyectos, a su vez es importante tener en cuenta que para la actualidad este tipo de viajes solo podrá ser realizado por cierta parte de la población mundial, en pocas palabras las personas con mayor cantidad de dinero del mundo .

El turismo espacial se está convirtiendo cada vez más en una realidad, gracias a los avances técnicos y tecnológicos de la actualidad, los cuales contribuyen a que sea más eficiente la creación de medios de transporte por los cuales los turistas puedan vivir esta experiencia inolvidable. Como complemento del turismo espacial, las entidades privadas que están invirtiendo dinero, para que la experiencia para los turistas sea aún más completa, es por eso que estas organizaciones están planteando la idea de crear establecimientos hoteleros en el espacio exterior, y así mismo llegar en un futuro a aumentar este tipo de actividades; ya realizando un enfoque a los destinos que se tienen en cuenta por implementar, se puede evidenciar que los que más llaman la atención para los inversionistas y más importante aún para los turistas es la Luna y Marte, debido a esto en la actualidad algunas compañías como SpaceX en alianza con la NASA tienen dentro de sus ideas de negocios poder llegar a la realización de algunos de estos viajes y que no solo tenga como enfoque una investigación científica, sino que también sea un viaje en el que el turismo sea el eje principal. Por otro lado, a pesar de que Marte está siendo considerado como una buena idea de destino turístico, ha presentado muchos factores en contra ya que según investigaciones realizadas por diferentes tipos de empresas como la NASA y a su vez científicos, se evidencia que la vida en este lugar no prosperaría y por eso mismo no es recomendable que una persona lo escogiera como un destino (Stacey). Es por esto, que en la actualidad es muy difícil llegar a evidenciar algún tipo de idea en la que se pueda vender a Marte como un nuevo destino turístico, y por esta misma razón es que solo se está tomando a la Luna como el destino que en futuro no muy lejano va a poder recibir turistas.

Independientemente de los destinos innovadores que se están teniendo en cuenta actualmente, es importante resaltar que la opinión que más vale es la de los turistas y que por esto es que es necesario antes de realizar cualquier cambio que genere impacto, es importante siempre fijarse en los requerimientos y necesidades que tiene el cliente en este caso el turista para así mismo poder buscar la mejor solución, y ofrecer el mejor servicio para que así el cliente pueda llevarse una experiencia inolvidable.

Los inicios del turismo espacial comenzaron con el proyecto Mercury en el año 1958, utilizando los vastos recursos con los que contaba el gobierno de los Estados Unidos, uno de los importantes objetivos era comprobar los límites del cuerpo humano en el espacio, para obtener dicha información fueron lanzados al espacio animales y robots. 
El primer viaje tripulado fue el 12 de abril de 1961, el cosmonauta soviético Yuri Gagarin quien orbitó en torno a la tierra a bordo de una nave espacial Vostok. Tiempo después más exactamente el 5 de mayo de 1961 Alan Shepard Jr, fue el primer estadounidense en ser lanzado al espacio a bordo del Mercury Redstone 3, su vuelo duró alrededor de 15 minutos y fue llamado "Freedom 7" este evento fue presenciado por 45 millones de telespectadores.

Entre los años 1961 y 1963, seis naves más tripuladas volaron al espacio como parte del proyecto Mercury, los tripulantes viajaban en cápsulas sin alas, que se soltaban del cohete y volvían a caer a la tierra, esta nave fue diseñada para poder soportar las temperaturas que se sufrían al entrar de nuevo a la atmósfera y para aguantar el amerizaje en el océano.

En el año 1969 más exactamente el día 20 de julio, Apolo XI, misión estadounidense, aterriza en la superficie lunar por primera vez en la historia del hombre, esta misión había partido 10 horas antes en Florida, ante millones de telespectadores a nivel mundial, y se oyeron palabras famosas del astronauta en cabeza de la misión Neil Armstrong: "Un pequeño paso para el hombre un gran salto para la humanidad".

El presidente de ese entonces, John F. Kennedy, fue la persona que inició la llamada "Carrera espacial americana" en el año 1961, el expuso ante el gobierno y explicó la necesidad de que porque el hombre tenía que llegar a la luna antes de acabar el siglo. Comenzaron los programas espaciales Mercury y Gemini que más tarde se unirían para formar el famoso proyecto Apolo, los astronautas se acostumbraron a vivir en la nave espacial e incluso fuera de ella. El cosmonauta soviético Aleksei Leonov fue la primera persona en la salir a la órbita en marzo de 1965. Las naves contaban con un módulo de mando, uno de servicio y un módulo lunar, el Apolo Xl logró aterrizar en la Luna, la misión de estos tripulantes era aterrizar en la Luna y conseguir muestras de la superficie lunar, no se sabía con certeza los efectos reales de la gravedad lunar en el comportamiento de los astronautas, respecto a su movilidad y mente, tampoco se sabía si volverían con algún tipo de contaminación.

Finalmente, Apolo Xl logró con éxito aterrizar sobre el módulo lunar llamado "Eagle" este nombre fue puesto en honor al ave representativa del país, las primeras palabras de Armstrong transmitidas en televisión fueron: "Estoy al pie de la escalerilla. Las patas del Águila sólo han deprimido la superficie unos cuantos centímetros. La superficie parece ser de grano muy fino, cuando se la ve de cerca. Es casi un polvo fino, muy fino. Ahora salgo de la plataforma."

Actualmente los Estados Unidos quieren regresar a la Luna y ya es un hecho oficial, el presidente Donald Trump afirmó ante la Directiva de Política Espacial 1, la cual autoriza a la Nasa liderar un programa innovador de exploración espacial para enviar astronautas a la luna y posteriormente a marte. En octubre del 2017, el vicepresidente de Estados unidos Mike Pence, dio las primeras pistas de los planes que buscan llevar al hombre de nuevo a la Luna, pero por el momento se desconocen muchos detalles para llevar a cabo esta misión. 
El objetivo de la nueva misión será construir en la Luna una base que sirva de conexión y laboratorio para otras misiones, una de ellas Marte, y extraer materiales que sirvan o puedan ser usados en nuestro planeta. El gobierno quiere que los astronautas obtengan más experiencia en exploraciones más allá de la Estación Espacial Internacional, para preparación de misiones más complejas y con mayor duración.

Hay un sector de especialistas que rechaza la idea de volver a la luna ya que el presupuesto se podría enfocar más en viajes a Marte y así no retrasar más otros proyectos. Se cree que ir a la Luna es más un tema competitivo ya que actualmente países como China, Japón, India, Rusia, compañías privadas como Blue Origin, Boeing y SpaceX buscan llegar a la Luna.

Rusia es otra de las potencias mundiales que no se quiere quedar atrás en los viajes al espacio, en el 2015, Rusia hizo 29 lanzamientos de impulsores especiales 18 de estos lanzamientos se realizaron desde el cosmódromo de Baikonur en la ciudad de Kazajistan, otros tres desde el puerto espacial de Kourou en Guyana, uno desde la región rusa Oremburgo y los otros 7 desde el cosmódromo del ministerio de defensa ruso en Plesetsk, esto lanzamientos superan considerablemente la cantidad de lanzamientos realizados por Estados Unidos, ya que durante el mismo plazo Estados unidos envió 19 cohetes al espacio de los cuales 17 fueron lanzados desde cabo Cañaveral y los otros dos desde la base Vandenberg, informó la corporación espacial rusa Roscosmos.

Durante el año 2015 se realizaron 86 lanzamientos de cohetes con fines espaciales, el cosmódromo de Baikonur fue la instalación espacial que realizó más lanzamientos al espacio con un total de 18 cohetes enviados al espacio. Mientras tanto China, tiene en sus planes explorar ya alunizar en la cara oculta de la Luna y posteriormente visitar Marte, aunque China por el momento no descarta una misión tripulada antes de 2022 a la Luna, el presupuesto de China ha estado en crecimiento a un ritmo de $10 \%$ cada año en la última década, se estima que 2.000 millones de dólares anuales.

Frente al estancamiento de los fondos para la NASA, China tomó posesión en el tablero espacial. Hace más de cuarenta años China envió por primera vez al espacio un satélite. Desde el año 2003 China no ha puesto a ningún Taikonauta (así se le llama a los astronautas de China).

Después de tanto tiempo China quiere volver a la Luna, pero quiere alunizar en su cara oculta, algo que es un poco más riesgoso y sin precedentes, ninguna de las misiones registradas actualmente a realizado este tipo de alunizaje, las autoridades científicas prometieron llegar a la cara oculta antes del año 2020, pero seguramente hagan el lanzamiento de la nave Chang'e- 4 en el 2019. 


\section{Metodología}

Teniendo en cuenta la revisión literaria, actualmente la actividad turística se encuentra en cambios constantes y se encuentra sumergida en propuestas innovadoras, es por esto que se puede evidenciar que actualmente debido a el nivel de demanda de pasajeros, es necesario que también el sector aeronáutico vaya innovando, por eso es que surgió la idea de buscar nuevos destinos turísticos que puedan satisfacer las necesidades de los viajeros.

Es importante tener en cuenta que, para poder llegar a escoger nuevos destinos, se debe pensar no sólo a nivel regional sino a nivel internacional, e incluso fuera del planeta, de igual manera también es importante estar en actualidad frente a los requerimientos que tienen los pasajeros, así mismo poder ofrecer destinos únicos y excepcionales para ellos. Es por eso que una idea que está llamando mucho la atención en este sector, es la realización de los primeros viajes fuera del planeta Tierra, en los cuales los turistas van a poder contar con la oportunidad de poder tener una experiencia única, y conocer un nuevo mundo que a su vez los puede llenar de muchos conocimientos.

Es por estas razones que una idea que está llamado la atención en el sector aeronáutico es poder realizar viajes tanto a la Luna como también a Marte, para así poder lograr las nuevas tendencias innovadoras que tanto se buscan y así poder cumplir con todas las expectativas que se generan y a su vez poder estar actualizado con el mundo de hoy. También se evidencia que ciertas compañías están trabajando en la realización de estos proyectos y que cada una busca ser conocida por las diferentes ideas innovadoras que les ofrecen a sus clientes.

Las compañías que evalúan cada una de estas ideas son:

SPACEX: Es una compañía privada estadounidense fundada por Elon Musk, también fundador de Tesla. (Antón, 2017). Esta empresa es conocida dentro del mundo aeronáutico por sus ideas en cuanto al diseño de naves y cápsulas para los viajes espaciales. El proyecto más importante de SpaceX hasta la fecha es el cohete Falcon Heavy, que cuenta con una potencia y una capacidad de carga superior a la del Saturno V (Frutos, 2018). Con respecto a destinos turísticos tiene pensado la realización de un viaje en el que dos pasajeros van a poder realizar un viaje alrededor de la luna.

VIRGIN GALACTIC: Tiene como eje principal la realización de viajes suborbitales, es decir que, durante el trayecto no se sobrepasará el límite del espacio ni se podrá realizar la vuelta completa hacia el planeta Tierra, pero durante el trayecto los turistas podrán realizar experimentos científicos, y así mismo, poder vivir la experiencia de sentir la gravedad cero.

Según Sir Richard Branson: "Ha sido genial ver a nuestra bonita nave volar por el aire y compartir este momento con el equipo" (ciencia, 2018), lo cual indica el crecimiento de constante rapidez de esta compañía en el sector. 
BLUE ORIGIN: Cuenta con el prototipo de una cápsula suborbital tripulable, la cual está conformada por múltiples ventanas en las que los turistas pueden observar la inmensidad tanto del espacio como del planeta Tierra, también cuenta con un valor agregado: los pasajeros van a caer sobre la superficie de la Tierra con un paracaídas, es importante aclarar que esta cápsula tiene capacidad para 6 pasajeros. Jeff Bezos, fundador y director de Amazon, tiene su propia compañía espacial, Blue Origin y recientemente ha anunciado sus planes de crear una nave de carga para hacer envíos a la Luna. Amazon, pero en el espacio. (Marín, 2018)

ORION SPAN: La idea que tiene esta compañía, es realizar un vuelo en el cual los pasajeros podrán llegar a la estación espacial aurora y contarán con actividades como cultivar alimentos, realizar experimentos científicos, sentir la gravedad cero y así mismo poder ver las diferentes auroras, este vuelo está diseñado para únicamente 6 pasajeros.

Según Orion Span y su sitio web: “Aurora Station será el primer hotel espacial de lujo del mundo en órbita a 200 millas sobre la superficie de la Tierra”. (WEB, 2017).

\section{Resultados Preliminares}

De acuerdo con la revisión literaria y la metodología expuestas, se evidencia que todas las organizaciones y compañías ya mencionadas, están dispuestas a comenzar con las ideas y proyectos planteados, tomando como propósito principal el de poder llevar al hombre nuevamente a la Luna, pero esta vez con fines turísticos. Algunos de los proyectos a realizar son los siguientes:

La NASA es una de las organizaciones que ha mostrado cierto interés y una gran disposición con respecto al sector turismo-comercial, pues están dispuestos a contratar compañías externas y privadas que suplan con algunos astronautas, medios tecnológicos y demás. A su vez, están aportando técnicas expertas con respecto al campo científico, buscan asociarse con compañías interesadas en el turismo espacial y así empezar a trabajar con ellas.

La empresa SpaceX ya tiene al humano número 25 que viajará a la Luna en el 2023 a bordo del BFR o como es mejor conocido el Big Falcon Rocket, se trata del japonés multimillonario Yusaku Maezawa, fundador de Zozotown, el minorista de línea de ropa más grande de Japón.

La cantidad que pagó Maezawa es significativa ya que lo que se pretende poder hacer es ver de cerca el satélite natural de la Tierra, pero este no alunizara. Cabe aclarar, que antes del viaje programado para el 2023, Elon Musk, fundador de Space X, ya había confirmado a dos pasajeros para realizar el vuelo a finales del 2018 hacia la Luna en el Falcon Heavy, pero esto se canceló ya que decidió darle prioridad al BFR. (Barco, 2018)

La empresa privada Boeing se ha encargado de construir una cápsula llamada Crew Space Transportation (CST-100 Starliner) y los primeros vuelos tripulados serán a bordo de esta cápsula y de la nave Crew Dragon de la empresa Space X. La cápsula pretende transportar pasajeros a la Estación Espacial Internacional (ISS) y a otras estaciones privadas como a la 
futura Estación Comercial Space Station, se realizará en el año 2019 y tendrá un costo aproximado de $\$ 80$ millones de dólares por asiento. La cápsula deberá estar diseñada para su compatibilidad con el cohete Falcon 9, Delta IV y el atlas V la cual transportará un máximo de 7 personas. Mientras se encuentra en la órbita tendrá una duración de siete meses y pretende ser utilizada para realizar hasta diez misiones más. (XATAKA, 2018)

Blue Origin, planea vender boletos de vuelos suborbitales para el año 2019, estos vuelos se realizarán a bordo del cohete New Shepard, se trata de un cohete propulsor reutilizable y una cápsula con capacidad para máximo 6 pasajeros, los primeros tripulantes de prueba serán empleados de la compañía y luego el New Shepard podrá recibir turistas espaciales de forma comercial. El vicepresidente de la compañía, Rob Myerson, dijo que en el 2019 se tiene prevista la venta de boletos oficialmente y el precio estimado se encuentra en un rango de $\$ 50.000 \mathrm{y}$ \$250.000 dólares. Sin embargo, su competidor directo, Virgin Galactic lleva años vendiendo boletos para vuelos suborbitales y aún sigue probando su nave espacial VSS Unity pues esta es la nave en la que planean realizar estos vuelos. (GIZMODO, 2018)

La última y más reciente propuesta es de Jeffrey P. Bezos, integrante de la compañía Blue Origin ha estado observando cómo se mueve el sector comercial y científico. Este propone desarrollar una nave espacial lunar donde supone llegar a un cráter en la parte sur, pues allí se ha evidenciado agua y también energía solar lo cual es un aspecto muy positivo para las personas que aterricen en esta zona. Esta propuesta llamó mucho la atención de Elon Musk, pues por su parte también tiene planeado enviar a un humano hacia la Luna y este no iría con fines científicos, ni a realizar investigaciones si no iría con el fin de realizar turismo espacial.

Ya que las personas que irían al espacio no son personas capacitadas y tampoco se desarrollan en el campo científico, por lo tanto, la agencia espacial ha decidido enviar con ellos a dos astronautas que sean capaces de llevar a cabo la misión de manera satisfactoria y junto con ellos también irían cuatro personas que conformarán el equipo de la tripulación, pues en este caso es necesario el conocimiento turístico que ellos poseen. (Davenport, 2017) 


\section{Conclusiones}

Finalmente, se demuestra que la posible adaptación de las nuevas tecnologías en los diferentes destinos creará una visión moderna del turismo donde el pasajero disfrutará de los nuevos destinos deseados. De acuerdo con la revisión literaria y con la metodología expuestas, se evidencia que organizaciones como la NASA y empresas como SpaceX, Virgin Galactic, Blue Origin y Orion Span, están impulsando a la realización de diferentes viajes con fines turísticos hacia la Luna y hacia Marte, a pesar de que ha sido una idea que se ha tenido desde hace mucho tiempo, es hasta ahora, que gracias a los avances tecnológicos, se puede evidenciar los posibles resultados de dicha idea; la de poder implementar un turismo espacial, y así mismo poder llevar consigo tripulación y turistas.

Es el sector comercial el que también está interesado en poder enviar a las personas al espacio, pues es algo con lo que el ser humano sueña, además es importante para ofrecer nuevas soluciones a las demandas de los clientes potenciales antes que los demás, en sentido amplio el eje de la innovación en el destino es el "cliente" en ellos la innovación supondría la incursión de la población en la planificación turística y de transporte aéreo del destino el espacio, pero desafortunadamente conlleva tiempo, presupuesto, investigaciones científicas y otros aspectos importantes a tener en cuenta los cuales no facilitan el próspero desarrollo de esta actividad. Aun así, el avance de las nuevas tecnologías de la mano de estas compañías, podrá generar un futuro enriquecedor en el sector permitiendo el posicionamiento del mercado turístico y el transporte aéreo fuera del planeta tierra. 


\section{Referencias}

ANTÓN, P. (2017). 20 MINUTOS.

Obtenido de https://www.20minutos.es/noticia/3255064/0/spacex-quien-es-elon-muskmultimillonario/

CIENCIA, A. (2018). ABC CIENCIA.

Obtenido de https://www.abc.es/ciencia/abci-virgin- galactic-eleva-35-kilometros-naveturismo-espacial-201805301330_video.html

FRUTOS, A. M. (2018). COMPUTER HOY.

Obtenido de https://computerhoy.com/noticias/life/que-es-spacex-77213

LÓPEZ, M. I. (2018). NASA EN ESPAÑOL.

Obtenido de https://www.lanasa.net/news/reportajes-especiales/45-aniversario-de-la-llegadadel-hombre-la-luna/

MARÍN, E. (2018). GIZMODO.

Obtenido de https://es.gizmodo.com/blue-origin-la- compania-espacial-de-jeffbezos-planea-1826389324

REI, M. F. (2016). MUY HISTORIA.

Obtenido de https://www.muyhistoria.es/contemporanea/preguntas-respuestas/cuando-sefundo-la-nasa-871501240289

WEB, O. S. (2017). ORION SAPN.

Obtenido de https://www.orionspan.com/

BBC. (2017). SPACEX ANUNCIA EL PRIMER VIAJE TURÍSTICO A LA LUNA PARA FINALES DE 2018.

Obtenido de https://www.bbc.com/mundo/noticias-39111530

INFOBAE. (2018). ASÍ ES LA NUEVA CÁPSULA ESPACIAL DE LA NASA CONSTRUIDA POR BOEING.

Obtenido de https://www.infobae.com/america/fotos/2018/08/03/asi-es-la-nueva-capsulaespacial-de-la-nasa-construida-por-boeing/

NATIONAL GEOGRAPHIC. (2010). EL PRIMER VUELO ESPACIAL TRIPULADO.

Obtenido de https://www.nationalgeographic.es/espacio/el-primer-vuelo-espacial-tripulado 
NMAS1. (2018). BLUE ORIGIN COMENZARÁ SU PROGRAMA TURISMO ESPACIAL EL PRÓXIMO AÑO.

Obtenido de https://nmas1.org/news/2018/06/26/blue-origin-turismo- 2019-ciencia

SOBRE HISTORIA. (2018). LA LLEGADA DEL HOMBRE A LA LUNA.

Obtenido de https://sobrehistoria.com/la-llegada-del-hombre-a-la-luna/

ROLDAN GÓMEZ, A. (S.F.). TURISMO ESPACIAL.

Obtenido de https://www.acta.es/medios/articulos/cultura_y_sociedad/050025.pdf

STACEY, A. (S.F.). MARTE NECESITA AGENTES DE BOLSA.

Obtenido de

http://www.dcne.ugto.mx/Contenido/MaterialDidactico/amezquita/Lecturas/Marte.pdf

HISPANTV. (2015). RUSIA VUELVE A LANZAR MÁS COHETES ESPACIALES QUE EEUU.

Obtenido de https://www.hispantv.com/noticias/ciencia-tecnologia/199090/rusia- eeuulanzamientos-cohetes-espaciales

LA VANGUARDIA. (2017). LA LARGA MARCHA DE CHINA HACIA LA LUNA. Obtenido de https://www.lavanguardia.com/internacional/20170409/421525581231/lunachina-carrera-espacial-larga-marcha.html

BARCO, L. D. (2018). HIPERTEXTUAL. Obtenido de SPACEX anuncia que el multimillonario Yuzaku Maezawa será el primer turista en viajar a la luna.:

https://hipertextual.com/2018/09/spacex-yusaku-maezawa-luna

XATAKA. (2018). LA PRIMERA MISIÓN DE SPACEX TRIPULADA POR ASTRONAUTAS DE LA NASA YA TIENE FECHA, JUNIO DE 2019.

Obtenido de https://www.xataka.com/espacio/primera-mision-spacex-tripuladaastronautas-nasa-tiene- fecha-junio-2019

GIZMODO. (2018). BLUE ORIGIN DICE QUE COMENZARÁN A VENDER BOLETOS PARA VIAJES ESPACIALES EN 2019. Obtenido de https://es.gizmodo.com/blue-origindice-que-comenzaran-a-vender-boletos-para-v-1827061692

DAVENPORT, C. (2017). THE WASHINGTON POST (WP COMPANY LLC).

Obtenido de https://www.washingtonpost.com/news/the-switch/wp/2017/03/02/an-exclusivelook-at- jeff-bezos-plan-to-set-up-amazon-like-delivery-for-future-human-settlement-of-the-

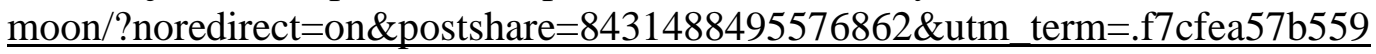






